\title{
Study in momentum space of phase-dependent effects on ionization of hydrogen atom interacting with short infrared laser pulses
}

\author{
Fréderic ONGONWOU ${ }^{1}$, Hugues Merlain TETCHOU ${ }^{2}$, Thierry Blanchard EKOGO ${ }^{3}$, \\ Bakari ABDOURAMAN ${ }^{4}$, and Moïse Godefroy KWATO \\ ${ }^{1}$ Universite des Sciences et Techniques de Masuku \\ ${ }^{2}$ University of Douala Faculty of Sciences \\ ${ }^{3}$ Université des Sciences et Techniques de Masuku \\ ${ }^{4}$ Universite catholique de Louvain Faculte des Sciences
}

September 25, 2021

\begin{abstract}
We examine above-threshold ionization spectra of model atomic hydrogen in short infrared laser pulses by solving the oneelectron time-dependent Schr "odinger equation in momentum space. To bypass the difficulty of solving the time-dependent Schr \" odinger equation with the interacting nonlocal Coulomb potential, we have recently formulated an alternative $\backslash$ emph $\{$ ab initio\} approach [Ongonwou et al. Annals of Physics $\{\backslash$ bf 375\}, 471 (2016)], which is relied on the expansion of the atomic wavefunction and the interacting nonlocal Coulomb potential on a discrete basis set of Coulomb Sturmians in momentum space. As far as short infrared laser pulses are concerned, we have numerically evaluated the photoelectron momentum distributions, angular distributions and bound states populations. The results obtained from our accurate new computationally method are compared against predictions of other time-dependent calculations in the literature. This new theoretical model shows its sensitivity to the carrier-envelope phase of the laser pulse and captures the left-right dependence of the emitted photoelectrons momentum and angular distributions. More precisely, short pulses manifest significant dependence of the differential ionization probability on carrier-envelope phase of the laser pulse and broken forward-backward symmetry in the angular distributions.
\end{abstract}

\section{Hosted file}

Tetchou_CEP_Effects_IJQC_2021.pdf available at https://authorea.com/users/437082/articles/ 538971-study-in-momentum-space-of-phase-dependent-effects-on-ionization-of-hydrogenatom-interacting-with-short-infrared-laser-pulses 